\title{
Kinetics of Degradation of Metribuzin in aqueous solution using Zero Valent Iron Nanoparticles
}

\author{
K’Owino Isaac O. ${ }^{1}$, Veronica A. Okello ${ }^{2}$ and Kevin Masika ${ }^{1}$ \\ ${ }^{1}$ Department of Pure and Appiled Chemistry P. O. Box 190 Kakamega, Masinde Muliro University \\ of Science and Technolog, Kenya. \\ ${ }^{2}$ Department of Physical Sciences, P. O. Box 136-90100, Machakos University, Machakos, Kenya.
}

\begin{abstract}
In this work, zero-valent iron nanoparticles (nZVI NPs) were synthesized in $\left(\mathrm{EW}^{-\mathrm{Fe}^{0}}\right)$ ethanol water mixed system and have been evaluated for degradation in aqueous solution of metribuzin. The batch experiments at varying solution $\mathrm{pH}$ showed the degradation efficiency of $93.22 \%$, $83.74 \%$ and $70.09 \%$ for the $\mathrm{pH}$ value 10, 7 and 4 respectively. Application of the varying dosage; $\left(2 \% \mathrm{Fe}^{\mathrm{o}} \mathrm{w} / \mathrm{v}, 4 \% \mathrm{Fe}^{\mathrm{o}} \mathrm{w} / \mathrm{v}, 6 \% \mathrm{Fe}^{\mathrm{o}} \mathrm{w} / \mathrm{v}\right)$ reflected an increasing trend as per the recorded degradation efficiency of $54.36 \%, 67.28 \%$ and $79.38 \%$ respectively. Scanning electron microscopy (SEM) and Energy Dispersive X-ray Analysis (EDXA) were used to determine surface morphology and crystallinity of the particles respectively. The batch kinetic tests gave kinetics of a pseudo-first order along with the rate constants, $\mathrm{K}_{\mathrm{obs}}$ of $0.2659 \mathrm{~h}^{-1}, 0.2320 \mathrm{~h}^{-1}$ and $0.2066 \mathrm{~h}^{-1}$ after incubating $6 \%$ $\mathrm{Fe}^{\mathrm{o}}$ w/v with $10 \mathrm{ppm}, 15 \mathrm{ppm}$ and $20 \mathrm{ppm}$ of Metribuzin respectively. The residual metribuzin was monitored in the solution by using of high performance liquid chromatography (HPLC) and UV Spectrophotometer. Thus, we present nZVI as an alternative rapid method for the detoxification of metribuzin.

[DOI: 10.22401/JNUS.21.2.01]
\end{abstract}

Keywords: Metribuzin, Degradation, Zero Valent Iron Nanoparticles, pseudo-first order.

\section{Introduction}

The application of Metribuzin [4-amino -6-(1,1-dimethylethyl)-3-(methylthio)-1,2,4triazin-5(4H)-one] herbicide to soils is for controlling the broadleaf and grasses weeds in potatoes, asparagus, tomatoes, soybeans and sugarcane [1]. It is considered to be of short to moderate persistence in soils, with a half-life of between 5 and 50 days. It has low adsorption coefficient $\left(1.12-1.25 \mathrm{~mL} \mathrm{~g}^{-1}\right)$ and high water solubility $\left(1.05 \mathrm{~g} \mathrm{~L}^{-1}, 20^{\circ} \mathrm{C}\right)$. It is highly toxic with an $\mathrm{LD}_{50}$ of 150 to $250 \mathrm{mg} / \mathrm{kg}$ in mice [2]. Due to its high solubility in water (1200 $\left.\mathrm{mg} \mathrm{L}^{-1}\right)$, Metribuzin is prone to runoff into surface waters. Its direct application or accidental discharge to water courses, vapor drift and spray, results too in contaminated surface waters [3]. Additionally it may precipitate and cause groundwater intrusions from treated lands [3].

Its degradation by microbiological and chemical processes often results in the formation of deaminometribuzin (DA), diketometribuzin (DK), deaminodiketometribuzin (DADK) and glycoside conjugates whose toxicity levels are yet to be determined. Thus, it has potential for groundwater contamination especially in environmental conditions that do not favour degradation [4] and poses a great risk to humans, terrestrial, and aquatic fauna and flora $[5,6]$.

A number of environmental remediation procedures have been employed in the treatment of metribuzin pollution. Examples include adsorption processes such as granular carbon [7] and preparation of magnetic switchgrass through the precipitation of iron oxide onto biochar [8]. The continuous contamination of ground water bodies and surface is still as a result of sources of nonpoint from agricultural surface runoff [9]. However, in these systems a lot of water is needed to use it by continuously passing the water through the system, where some of the adsorbed amounts may also get to be desorbed [10]. Phytoremediation, and microbial degradation have also been widely used. Even then, these methods are consuming more time, poor effectiveness, cumbersome and high cost needed in order to set up and run.

Currently, zero-valent iron nanoparticles have gained interest to eliminate an array of toxic contaminants in the environment. This is because these particles are more effective at 
degrading some contaminants because of their higher reactivity due to increased surface area [11]. Batch reactors study on the influence of amine buffers on carbon tetrachloride dechlorination by iron oxide magnetite has previously been reported [12]. The extensive application of nanozerovalent $\left(\mathrm{Fe}^{0}\right)$ by itself or coupled with other oxidizing agents in the detoxification of various pesticides has been reported thereby opening a research window for investigating the use of other nanoparticles in environmental remediation [13]. Herein the use of zero-valent iron nanoparticles in the degradation of metribuzin in butch studies is presented.

\section{Materials and Methods}

Metribuzin Standard (99.0\%), analytical grade was purchased from Dr. Ehrenstorfer GmbH (Augsburg, Germany). Acetonitrile (HPLC grade, $98 \%$ purity), Sodium Borohydride, Ethanol and Ferric Chloride Hexahydrate of analytical grade were obtained from Kobian Company Limited. The HPLC grade acetonitrile was used in Metribuzin analysis. All other chemicals were reagents of analytical grade. The solutions were prepared using double distilled water. Metribuzin standard solutions were prepared by dissolving in acetonitrile.

\section{Preparation of ZVI NPs}

$9.4575 \mathrm{~g}$ sodium borohydride $\left(\mathrm{NaBH}_{4}\right)$ was dissolved in in $0.1 \mathrm{M}$ sodium hydroxide $(\mathrm{NaOH})$ solution to give $0.25 \mathrm{M} \mathrm{NaBH}$ aqueous solution. Similarly, $12.1635 \mathrm{~g}$ of $\mathrm{FeCl}_{3} \cdot 6 \mathrm{H}_{2} \mathrm{O}$ was added into $100 \mathrm{~mL}$ of ethanol/water mixture to give $0.045 \mathrm{M}$ $\mathrm{FeCl}_{3} \cdot 6 \mathrm{H}_{2} \mathrm{O}$ solution. Stabilized nano zero valent iron (nZVI) nanoparticles (NPs) were synthesized by dropwise addition of $0.25 \mathrm{M}$ $\mathrm{NaBH}_{4}$ aqueous solution to Neon gas-purged $0.045 \mathrm{M} \mathrm{FeCl}_{3} .6 \mathrm{H}_{2} \mathrm{O}$ aqueous solution in the ratio $1: 1$ at temperature of $\left(23^{\circ} \mathrm{C}\right)$ along with magnetic stirring. The solution was stirred for (20 $\mathrm{min})$ and centrifuged for $(2 \mathrm{~min})$. Then acetone was used instead of the supernatant solution. Washing by acetone was to prevent the immediate rusting of (nZVI) during purification, which lead to product of a fine black powder after freeze-drying. The particles were further washed 3 to 4 times with $25 \mathrm{~mL}$ portions of absolute ethanol. All nZVI NPs were stored in a refrigerator at $\left(<4{ }^{\circ} \mathrm{C}\right)$.

\section{Degradation Experiments Optimal nZVI NPs Concentration}

Batch experiments were conducted in $100 \mathrm{~mL}$ serum bottles containing $50 \mathrm{~mL}$ of aqueous Metribuzin solution with an initial concentration of $15 \mathrm{mgL}^{-1}$. Optimal nZVI NPs concentration was established using $2 \% \mathrm{w} / \mathrm{v}$, $4 \% \mathrm{w} / \mathrm{v}, 6 \%$ and $8 \% \mathrm{w} / \mathrm{v}$ of ZVI NPs respectively.

\section{Optimal Metribuzin Concentration}

Similarly, varying concentrations of metribuzin (10 ppm, $15 \mathrm{ppm}$ and $20 \mathrm{ppm}$ ) were used with optimal percentage of $8 \% \mathrm{w} / \mathrm{v}$ ZVI NPs. The bottles were fitted by using Teflon Mininert valves and the mixture was stirred on a proper shaker by $100 \mathrm{rpm}$ at $25^{\circ} \mathrm{C}$. Then solution was put into centrifuge device and run for $10 \mathrm{~min}$ with high speed centrifugation. Then the supernatant analyzed to quantification of metribuzin while residue was subjected to further physical chemical characterization.

At pre-determined time, $2 \mathrm{~mL}$ of the aqueous sample was withdrawn and centrifuged at $3000 \mathrm{rpm}$. Residual metribuzin concentration was analyzed using Shimadzu UV-Vis Spectrophotometer (Model 1800).

Control experiments consisted of similar reaction mixtures without nZVI NPs under identical conditions. To assure data quality, all of the experimental points were run in triplicates.

\section{pH effect}

The $\mathrm{pH}$ effect of the solution for Metribuzin destruction has monitored for further study. Samples of the Metribuzin solution were modified with suspension nanoparticles at initial values of $\mathrm{pH}(4,7,10)$, respectively. The $\mathrm{pH}$ of solution was set with $0.1 \mathrm{~N} \mathrm{HNO}_{3}$ and $0.1 \mathrm{~N} \mathrm{NaOH}$. Then the solutions were allowed to react for 6 hours.

\section{Characterization of Nanoparticles}

The characterization of solid-phase (nZVI NPs) was carried out by using the (SEM) that is equiped with energy dispersive X-ray analysis system (EDAX) (model: CARLZEISS EVO MA 15). The fresh nZVI NP was dried at $\left(25^{\circ} \mathrm{C}\right)$ in vacuum for $(60 \mathrm{~min})$ before scanning. 


\section{Analytical methods \\ HPLC Analysis}

HPLC analysis has performed by the following conditions: Shimadzu (LC-20AT) fitted with Shimadzu prominence (SPD-20A) detector of UV/visible, degasser (DGU-20A prominence, Shimadzu) and phenomenex 00 G-4420-E0 (HyperClone 5u BDS C-18 130A, 5 micron, $250 £ 4.60 \mathrm{~mm})$ column. Mobile phase is (acetonitrile:water 1:1) with rate of flow $\left(0.7 \mathrm{~mL} \mathrm{~min}^{-1}\right)$, the wavelength of detector is $254 \mathrm{~nm}$ and the volume of injection is $20 \mu \mathrm{L}$. The experiment was run with triplicate. The preparation of mobile phase was achieved with washing the bottles $(500 \mathrm{~mL})$ and then dried in drier for one hr, then filled with $500 \mathrm{~mL}$ mixture 1:1 of acetonitrile (HPLC grade) and water (double distilled). Finally, the mixture was degassed for $1 / 4$ hours before the pump (Shimadzu A VP LC-10AT) is running. The time of retention for Metribuzin and its metabolites was identified by using the standards reference. Further analysis was done using Shimadzu UV vis Spectrophotometer 1800 for quality assurance of data.

\section{Results and Discussion}

Characterization of Zero Valent Iron Nanoparticles

Analysis of surface changes before and after degradation experiments provided morphological and chemical changes on nZVI NPs. Fig.(1 a) and Fig.(1b) compares the SEM images of freshly prepared $\mathrm{Fe}^{0}$ nanoparticles and those particles after incubation with Metribuzin. The surface morphology images show discrete $\mathrm{Fe}^{0}$ nanoparticles in the range of microparticles $(100 \mu \mathrm{m})$. This was attributed to the agglomeration of the nanoparticles to form microparticles displaying images whose scale falls outside the nanoscale range. The aggregating has derived from magnetic forces that existing within the nanoparticles.

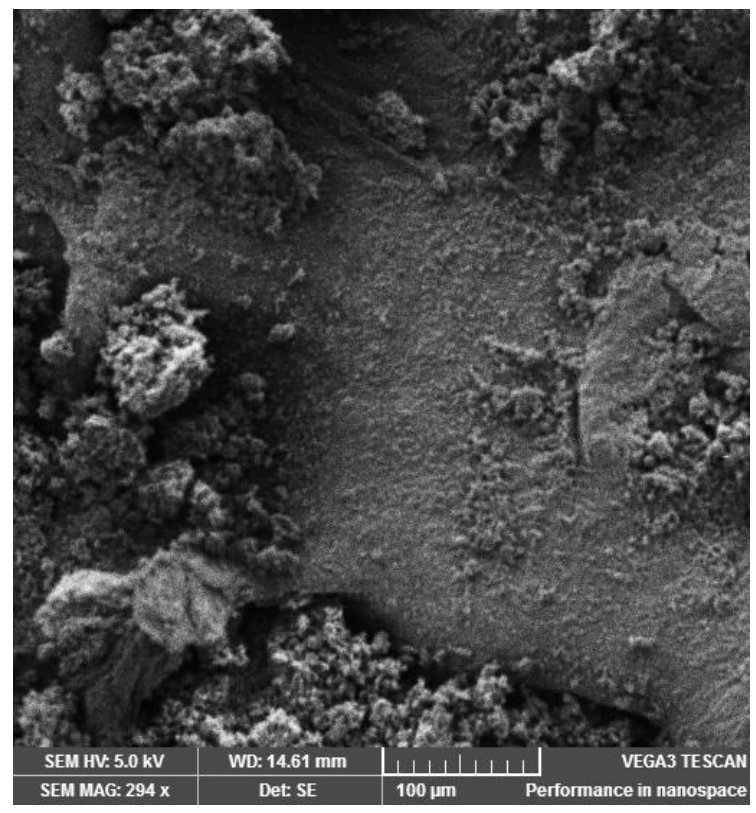

a

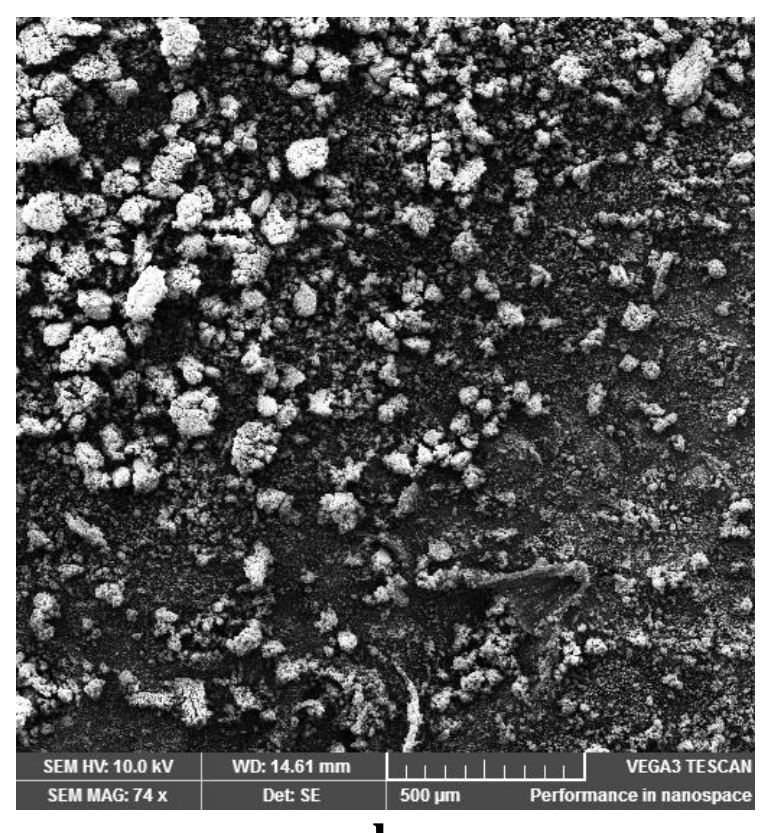

b

Fig.(1): The SEM analysis were carried out at $294 \mathrm{X}$ magnification at $\mathrm{kV} 5.0 \mathrm{~V}$ (a) for freshly prepared nanoparticles and (b) for the particles after incubation with metribuzin.

The morphological structure of Zero Valent Iron Nanoprticles changed after the interaction with metribuzin due to its reduction the possible subsequent oxidation of $\mathrm{Fe}^{0}$ to $\mathrm{Fe}^{3+}$ and $\mathrm{Fe}^{2+}$ as revealed by Energy Dispersive X-ray Analysis (EDXA) chemical analysis Fig.(2). 


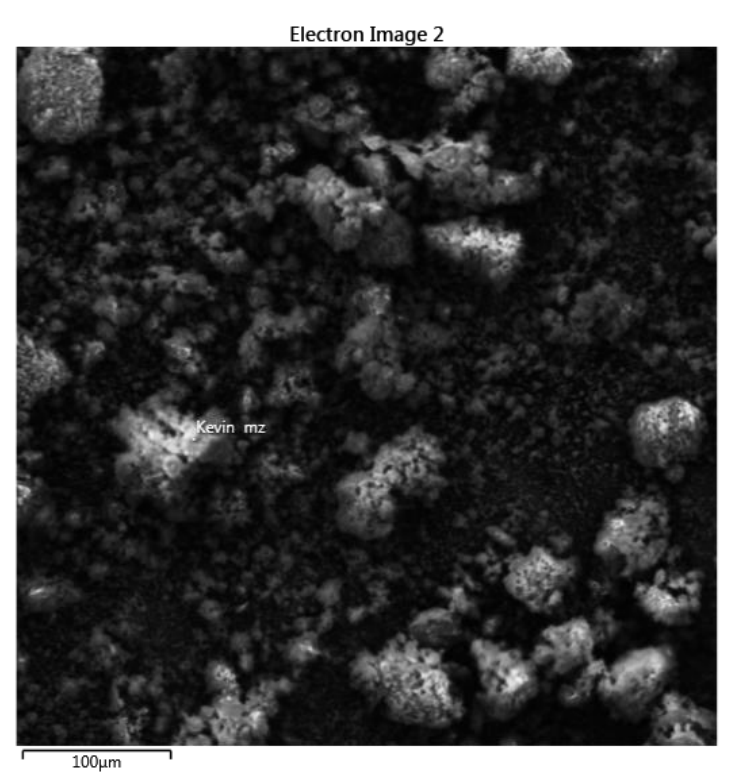

a

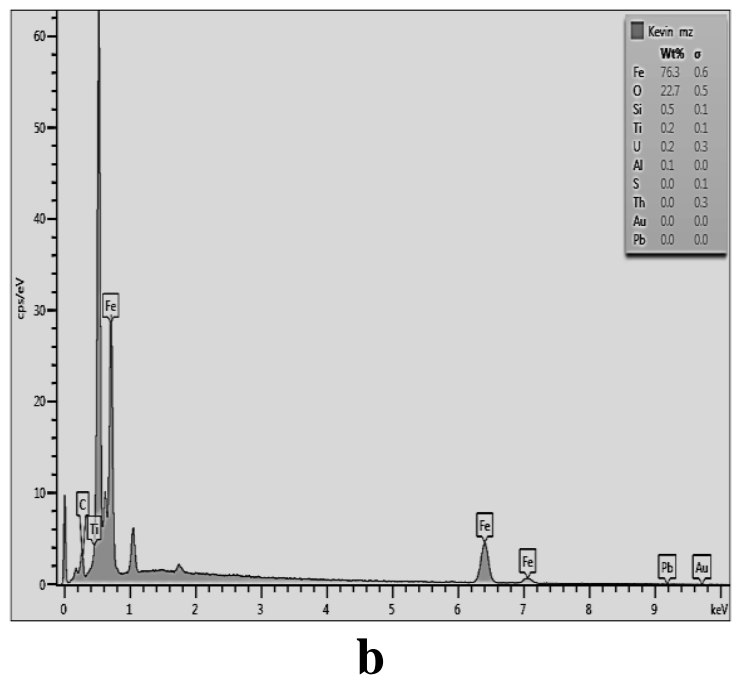

Fig.(2: a) Energy Dispersive X-ray Analysis (EDXA) image of ZVI NPs after incubation with Metribuzin. b) Chromatogram showing crystallinity of ZVI NPs.

In this case, the peaks displayed are $76.3 \%$ of iron and $22.7 \%$ of oxygen, $0.5 \%$ of silicon, $0.2 \%$ of uranium, $0.1 \%$ of tin, 0.2 and $0.1 \%$ of aluminium Fig.(2b). The intense photoelectron peaks of $\mathrm{Fe}$ after exposure is as a result of the formation of the dominant oxidation state of $\mathrm{Fe}^{3+}$ oxides and hydroxides accompanied with similar $\mathrm{Fe}^{2+}$ compounds

Previous studies [14] have shown that water (equation 1) and residual oxygen served as electron receptors thereby accounting for optimal reaction conditions at low $\mathrm{pH}$ (equation 2).

As such, the intense $\mathrm{O}$ peak is possibly attributed to $\mathrm{O}$ in the iron oxide lattice, $\mathrm{O}$ in structural $\mathrm{OH}^{-}$and $\mathrm{O}$ in physisorbed water.

$$
\begin{array}{r}
\mathrm{Fe}_{(s)}^{0}+2 \mathrm{H}_{2} \mathrm{O}_{(l)} \rightarrow \mathrm{Fe}^{2^{+}}{ }_{(a q)}+\mathrm{H}_{2(g)}+2 \mathrm{OH}_{(a q)}^{-} \\
\ldots \ldots \ldots \ldots \ldots \ldots \ldots \ldots \ldots \ldots \ldots \ldots \ldots \ldots \ldots \ldots \ldots \ldots \ldots \ldots \ldots \\
2 \mathrm{Fe}_{(s)}^{0}+4 H^{+}{ }_{(a q)}+\mathrm{O}_{2(g)} \rightarrow 2 \mathrm{Fe}^{2^{+}}{ }_{(a q)}++\mathrm{H}_{2} \mathrm{O}_{(l)}
\end{array}
$$

\section{Degradation Studies of Metribuzin}

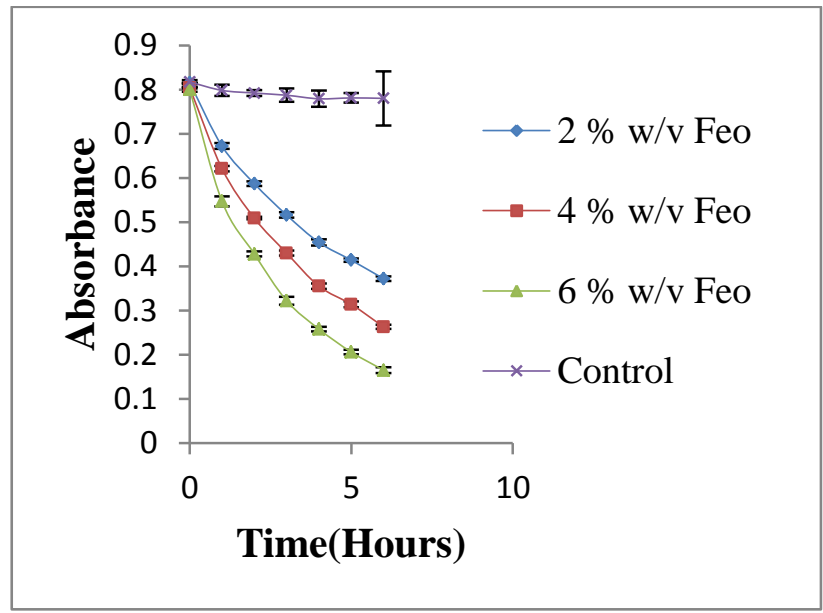

Fig.(3): Degradation of 15 ppm of Metribuzin using varying dosage of NZVI NPs.

The effect of $\mathrm{Fe}^{0}$ dosage using three different concentrations was investigated. In Fig.(3) it can be seen that the elevation of $\mathrm{Fe}^{0}$ concentration greatly enhanced the degradation of Metribuzin. In this study, the degradation rate increased when the concentration of $\mathrm{Fe}^{0}$ nanoparticles applied was increased from $2 \% \mathrm{w} / \mathrm{v}$ to $6 \% \mathrm{w} / \mathrm{v}$ respectively.

By observing the results, the degradation efficiency reached $79.38 \%, 67.28 \%$ and $54.36 \%$ when treated with $6 \%$ w/v $\mathrm{Fe}^{0}, 4 \%$ $\mathrm{w} / \mathrm{v} \mathrm{Fe}^{0}$ and $2 \% \mathrm{w} / \mathrm{v} \mathrm{Fe}^{0}$ respectively for an incubation period of 6 hours. In addition, the results show that degradation rate of Metribuzin was rapid at the start of the reaction and then eventually slowed down gradually as the reaction proceeded. This clearly indicates that the degradation rate was significantly dependent on the concentration of nZVI NPs. The blank experiment without nanoparticles did not show significant degradation of Metribuzin in relation to sample experiments. 


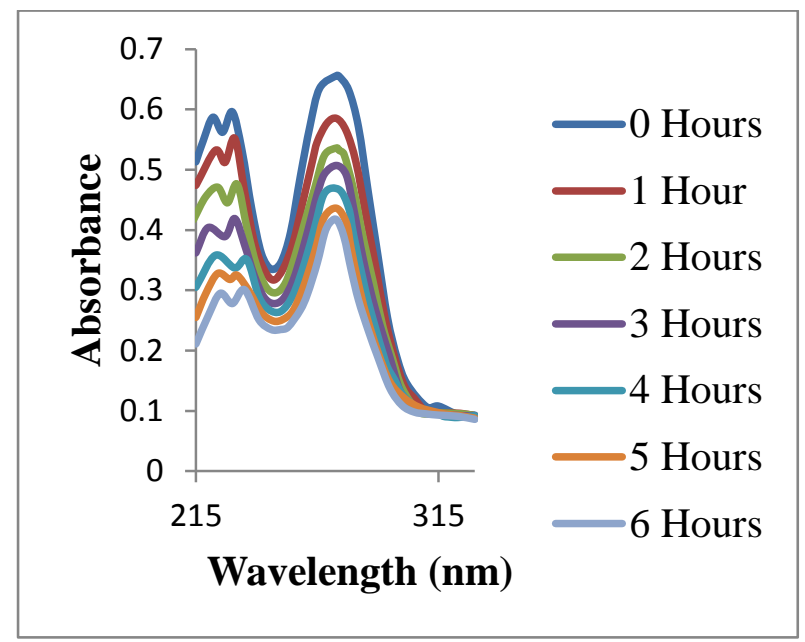

a

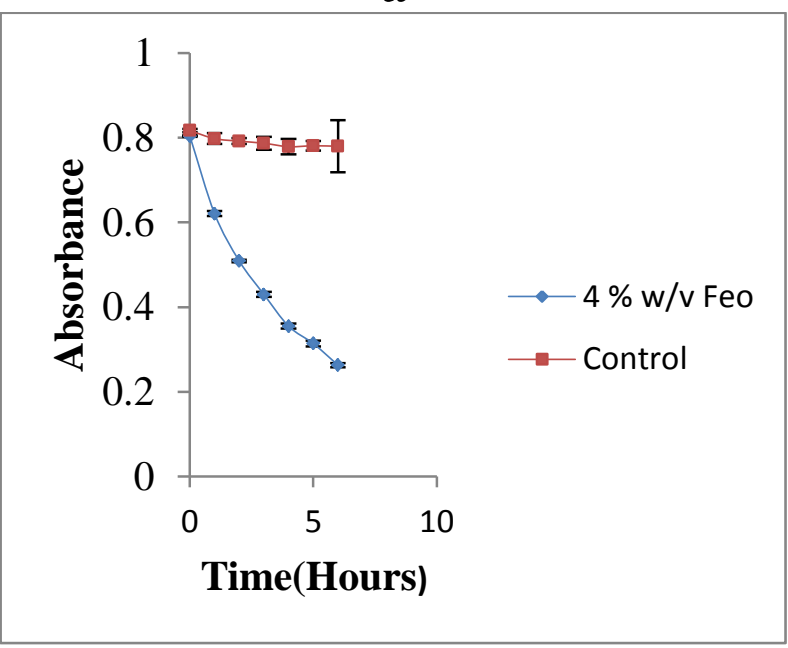

b

Fig.(4): (a) and (b) shows the change in concentration of 15 ppm of metribuzin as a function of time on incubation with $4 \%$ nZVI NPs for 6 hours.
Metrubuzin is UV activate with an absorbance maximum at $\approx 291 \mathrm{~nm}$ Fig.(4a). The change in absorbance was measured at $291 \mathrm{~nm}$ Fig.(4b). The results Fig.(4a) and Fig.(4b) demonstrate that $67.28 \%$ degradation of Metribuzin was achieved in 6 hours. In this case, control experiments carried out in the absence of nZVI NPs showed no appreciable loss Fig.(4b). Experiments were carried out (data not shown) to see the effect of change in the initial volume, in which the samples were withdrawn initially at zero minute and then directly after (3 hours). The observations indicated that the volume alteration on the reaction rate has no significant effect. For each experiment, the rate constant of degradation for the compound was calculated from the linear regression of the natural logarithm plot for the compound concentration as a function of time of incubation.

\section{Optimal degradation studies of Metribuzin}

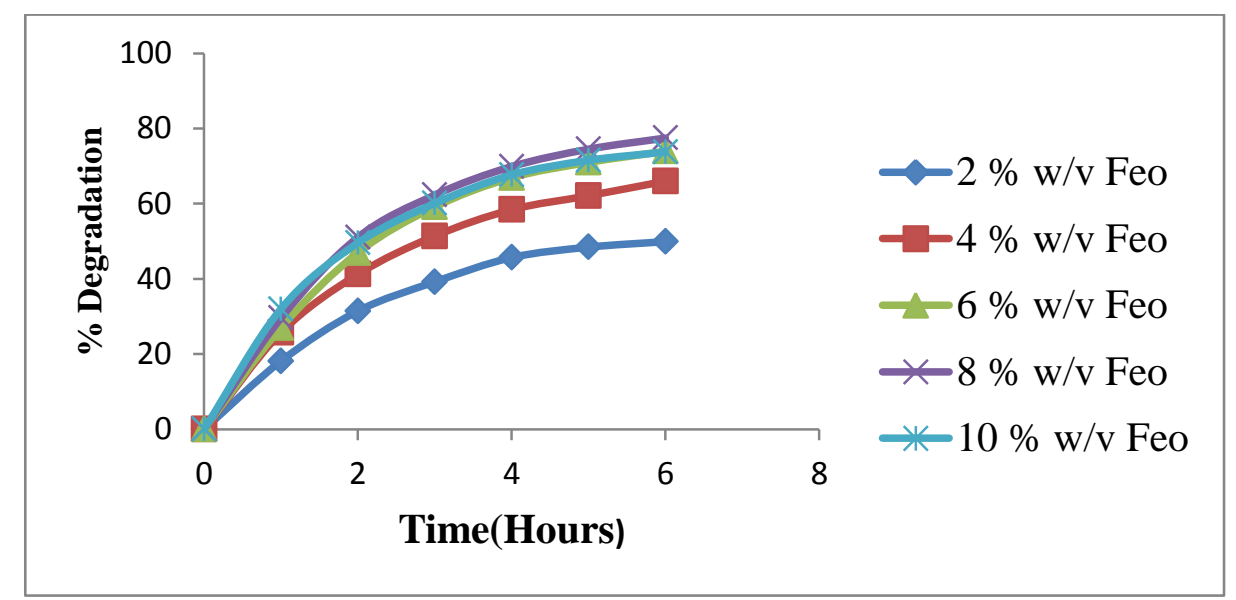

Fig.(5): Determination of optimum degradation of Metribuzin using varying the dosage of $\mathrm{Fe}^{\mathrm{O}}$ nanoparticles. 
The effect of $\mathrm{Fe}^{0}$ dosage was investigated for the degradation of $10 \mathrm{ppm}$ metribuzin using varying concentrations of ZVI NPs in the range of $2 \% \mathrm{w} / \mathrm{v} \mathrm{Fe}^{0}$ to $10 \% \mathrm{w} / \mathrm{v} \mathrm{Fe}^{0}$ for 6 hours. From the results in Fig.(5), the study showed that there was an increase in the degradation rate of metribuzin with the increased $\mathrm{Fe}^{0}$ dosage. However, the rate increased gradually from $2 \% \mathrm{w} / \mathrm{v} \mathrm{Fe}^{0}$ to $6 \%$ w/v $\mathrm{Fe}^{0}$ dosage with no significant changes after $8 \% \mathrm{w} / \mathrm{v} \mathrm{Fe}^{0}$ dosage. Therefore, it was concluded that the optimal degradation of metribuzin under normal room temperature and pressure could be achieved when $8 \%$ w/v $\mathrm{Fe}^{0}$ of ZVI NPs was applied.

\section{The effect of $\mathrm{pH}$ on the degradation of Metribuzin}

The $\mathrm{pH}$ effect of solution on the process of degradation of Metribuzin has investigated by putting all parameters of other are constant, such as dosage of $\mathrm{Fe}^{0}(6 \% \mathrm{w} / \mathrm{v})$, temperature $\left(25^{0} \mathrm{C}\right)$ and reaction time (6 hour). The $\mathrm{pH}$ of the solutions was varied 4 to 10 . The observed Metribuzin degradation rate was rapid at $\mathrm{pH}$ solution 4.0 as shown in Fig.(6). Degradation rates were $93.22 \%, 83.74 \%$, and $70.09 \%$ for $\mathrm{pH} 4,7$ and 10 respectively. From these results, it is sufficient to conclude that the degradtion of metribuzin by nZVI NPs occurs better in an acidic medum than basic medium. This is in agreement with several studies [15-17], which have reported slow degradation of most pesticides using $\mathrm{Fe}^{0}$ with the increasing $\mathrm{pH}$ value of the reaction medium.

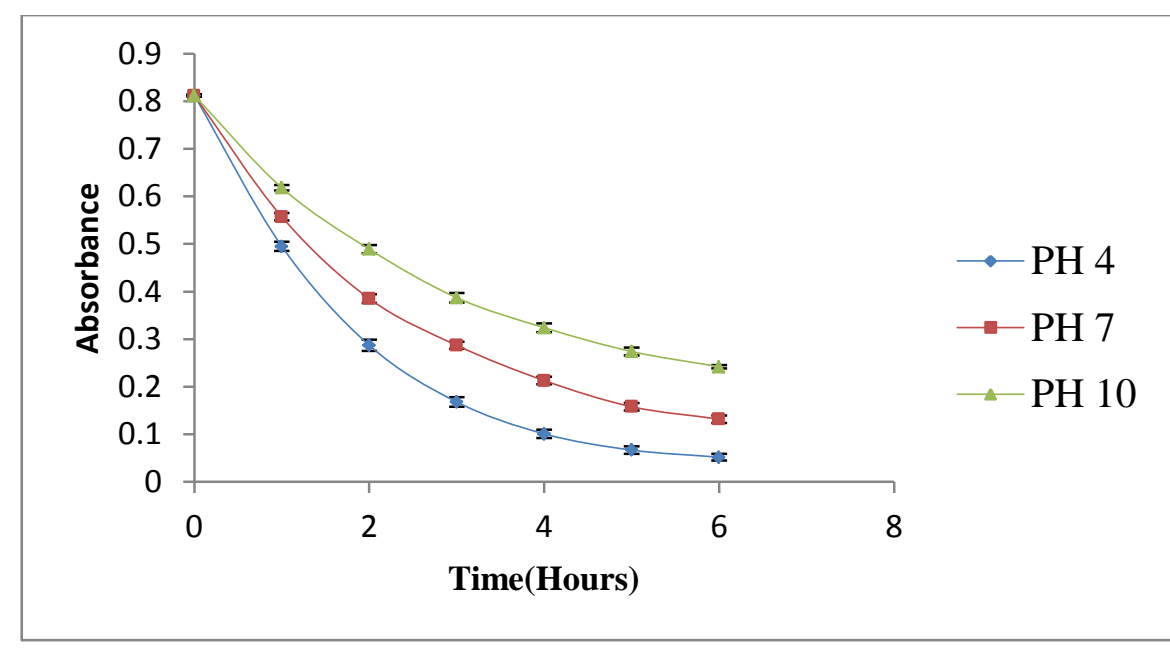

Fig.(6): pH effect on the degradation of Metribuzin.

This trend can be explained by the fact possible iron oxide formation on the $\mathrm{Fe}^{0}$ surface, which passivized iron surface, thus hindering the reactivity of nanoparticles surface. Therefore, the passivating layers would be removed by the low $\mathrm{pH}$ at surface of iron and rendering it can free for the fast reaction into the pesticides.

\section{Degradation Kinetics of Metribuzin}

Figs. (7) and (8) shows the linear regressions of natural logarithms of metribuzin initial concentrations $\mathrm{Ln}[\mathrm{C}]$ verses time showing first order Metribuzin degradation. The graphs gave a correlation coefficients of $\mathrm{R}^{2}=0.988, \mathrm{R}^{2}=0.9912$ and $\mathrm{R}^{2}=0.9917$ showing a good linearity.
In this case, the degradation of metribuzin was slow decreasing as the function of time. The degradation kinetics was explicated with pseudo-first order as shown below:

$$
\frac{d[c p p]}{d t}=-k_{o b s}[\mathrm{cpp}]
$$

Where the concentration of metribuzin is [cpp] in solution (aq.) at time (t) (min), time $\mathrm{K}_{\mathrm{obs}}$ is rate constant for the pseudo first order $(1 / \mathrm{min})$. Value $\left(\mathrm{K}_{\text {obs }}\right)$ is $0.1278 \mathrm{~h}^{-1}, 0.1815 \mathrm{~h}^{-1}$ and $0.2568 \mathrm{~h}^{-1}$ for $2 \% \mathrm{w} / \mathrm{v}, 4 \% \mathrm{w} / \mathrm{v}$ and $6 \%$ w/v of nZVI NPs. This showed that the rate of degradation was enhanced with increased concentration of the nZVI NPs. 


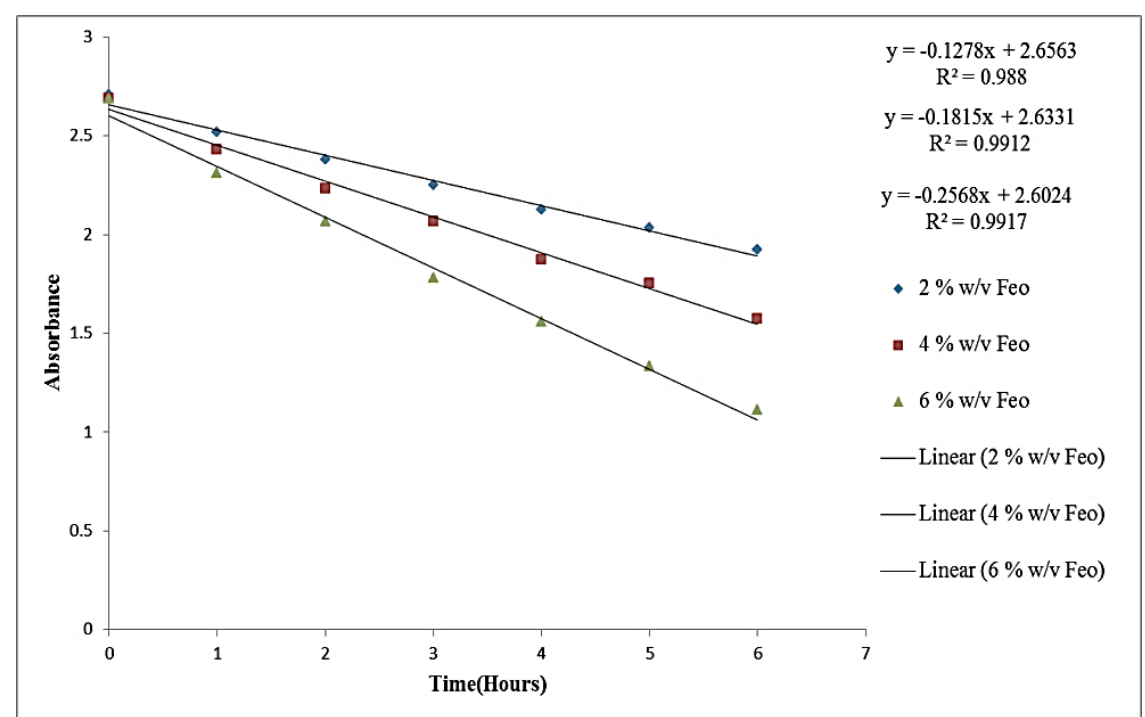

Fig.(7): Linear regressions of natural logarithms of Metribuzin initial concentrations Ln[C] verses time showing first order Metribuzin degradation.

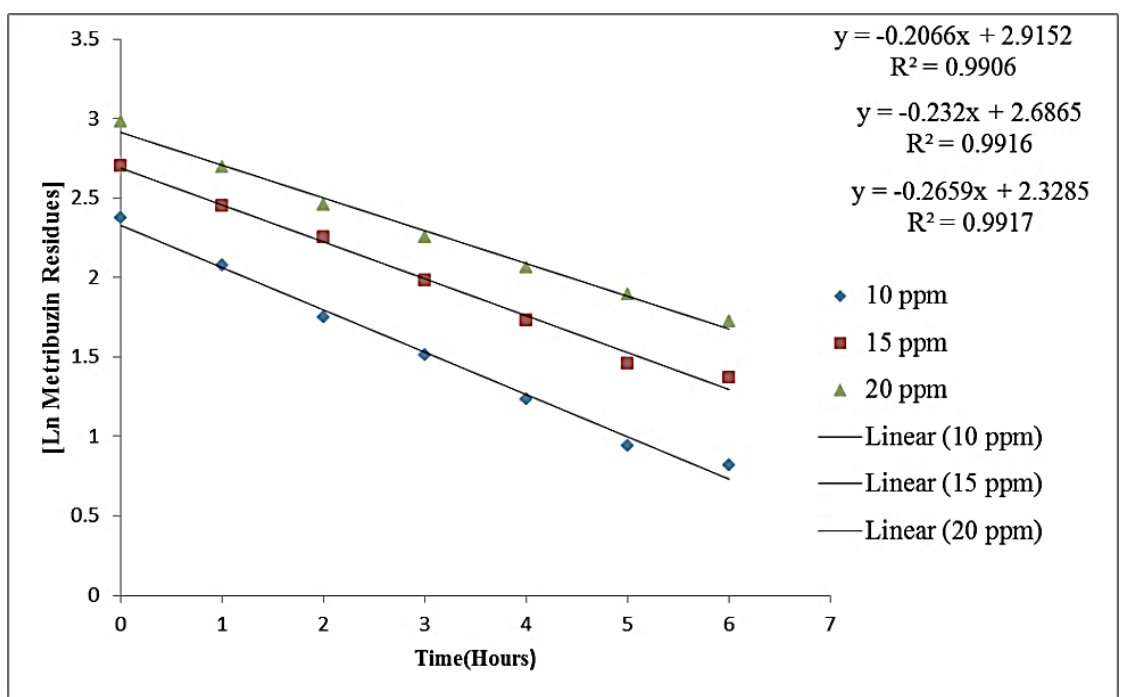

Fig.(8): Linear regressions of natural logarithms of Metribuzin initial concentrations Ln[C] verses time showing first order Metribuzin degradation. Different concentrations of 10ppm,

$15 p p m$ and 20ppm were incubated with $6 \% \mathrm{w} / \mathrm{v}$ of $\mathrm{nZVI}$ NPs for a period of 3 hours.

The initial effect of nMetribuzi concentration on the removal efficiency took place at concentrations of $\left(10,15,20 \mathrm{mgL}^{-1}\right)$ by using $6 \% \mathrm{w} / \mathrm{v}$ of (nZVI NPs). Kinetics of pseudo-first-order were generally used to describe the conditions of the reaction. The results from Fig.(8) confirm the kinetic model validity for Metribuzin with correlation coefficients of $\mathrm{R}^{2}=0.9917, \mathrm{R}^{2}=0.9916$ and $\mathrm{R}^{2}=0.9906$ respectively. The corresponding values of $\mathrm{k}$ are $0.2659 \mathrm{~h}^{-1}, 0.2320 \mathrm{~h}^{-1}$ and $0.2066 \mathrm{~h}^{-1}$ for initial concentration of $(10,15$ and 20) $\mathrm{mgL}^{-1}$ respectively, which clearly indicate that the decrease in rate constant is with increasing concentration of Metribuzin.
The metribuzin herbicide molecular structure is quite complex and it contains four $\mathrm{N}$ atoms which has state of oxidation (2), consequently it may participate in reduction reactions, in the presence of protons. $\mathrm{N}$ atoms of triazinon ring is sensible to the reaction of reduction. The Metribuzin degradation is depicted by azomethine protonated bond before the processes of transfer of electron. Protons attack on position 1 or position 2 of triazinon ring. Both azomethine reduction bonds (2-3,1-6) accompany by the equilibria of acid/ base and process of hydration and dehydration. $\mathrm{N}$ atom is ejected as ammonium hydroxide, as well as hydroxylamine. 


\section{Conclusions}

This study showed that Zero Valent Iron Nanoparticles are capable of degrading Metribuzin. The $\%$ dosage of $\mathrm{Fe}^{0}$ nanoparticles showed a significant effect on the Metribuzin degradation at $\mathrm{pH} 4$. The optimum degradation was achieved after application of $8 \% \mathrm{w} / \mathrm{v}$ of $\mathrm{Fe}^{0}$ nanoparticles with the degradation efficiency of $77.45 \%$ with a pseudo-first order reaction. Further studies on the structure of the degradation products and application to real samples are currently ongoing.

\section{Acknowledgments}

This work was supported by the National Commission of Science, Technology and Innovation, Kenya, under Grant number (NACOST/RCD/ST\&I $6^{\text {th }}$ CALL MSc/088). We thank the University of Johanesburg for their assistance in the Characterization of Zero Valent Iron Nanoparticles. We extend appreciation to Agnes Muyale for their technical assistance with HPLC analysis.

\section{References}

[1] Papadakis E.N., Papadopoulou-Mourkidou E., Determination of metribuzin and major conversion products in soils by microwaveassisted water extraction followed by liquid chromatographic analysis of extracts. Journal of Chromatography A., 962, 9-20, 2002.

[2] Bleeke M. S., Smith M T., Casida J. E., Metabolism and toxicity of metribuzin in mouse liver. Pesticide Biochemistry and Physiology., 23 (1), 123-130, 1985.

[3] Maloschik, E., Ernst, A., Hegeduss, G., Darvas, B., Szekacs., A. Monitoring waterpolluting pesticides in Hungary. Microchem.J., 85, 88-97, 2007.

[4] Lechón Y., García-valcárcel I.A., Matienzo T., sánchez-brunete C., Tadeo I.J., laboratory and field studies on metribuzin persistence in soil and its prediction by simulation models. Toxicological and environmental chemistry., 63, 47-61, 1997.

[5] Morley N. J, Environmental risk and toxicology of human human and veterinary waste pharmaceutical exposure to wild aquatic host-parasite relationships,
Environmental Toxicology and Pharmacology., 27 (2), 161-175, 2009.

[6] Huertas-P'erez F. J., Iruela O. M., Garc' 1aCampa na M. A., Gonz'alez-Casado A., S'anchez-Navarro A., Determination of the herbicide metribuzin and its major conversion products in soil by micellar electrokinetic chromatography. Journal of Chromatography A., 1102, 280-286, 2006.

[7] Kumar Y. B., Singh N., Singh S. B., Removal of Atrazine, Metribuzin, Metolachlor and Alachlor by Granular Carbon. J. Environ. Anal. Toxicol., 3, 196, 2013.

[8] Essandoh M., Wolgemuth D., PittmanJr C. U., Mohan D., Mlsna T., Adsorption of metribuzin from aqueous solution using magnetic and nonmagnetic sustainable low-cost biochar adsorbents. Environmental Science and Pollution Research., 24 (5), 4577-4590, 2007.

[9] Henriksen T., Svensmark B., Juhler K. R., Analysis of metribuzin and transformation products in soil by pressurized liquid extraction and liquid chromatographictandem mass spectrometry. J. Chromatogrs. A., 957: 79-87, 2002.

[10] Scherer E. M., Wang Q. Q., Hay A. G., Lemley, A.T., The binary treatment of aqueous metribuzin using anodic fenton treatment and biodegradation. Archives of Environmental Contamination and Toxicology., 47, 154-161, 2004.

[11] Saleh N., Kim H. J., Phenrat T., Krzystof M., Tilton R., Lowry G., Ionic Strength and Composition Affect the Mobility of Surface-Modified $\mathrm{Fe} 0$ Nanoparticles in Water-Saturated Sand Columns. Environ. Sci. Technol., 42, 3349-3355, 2008.

[12] Danielsen K. M., Gland J. L., Hayes K.F., Influence of Amine Buffers on Carbon Tetrachloride Reductive Dechlorination by the Iron Oxide Magnetite. Environ. Sci. Technol., 39 (3) 756-763.

[13] Rani, M., Shanker, U., Degradation of traditional and new emerging pesticides in water by nanomaterials: recent trends and future recommendations. Int. J. Environ. Sci. Technol., 1-34, 2017. 
[14] Yuan-Pang Sun, Xiao-qin Lia, Jiasheng Cao, Wei-xian Zhang, H. Paul Wang (2006) Characterization of zero-valent iron nanoparticles. Advances in Colloid and Interface Science., 120, 47-56, 2006.

[15] Walker, A., Jurado-Exposito, M., Bending, G. D., Smith, V. J. R., Spatial variability in the degradation rate of isoproturon in soil. Environ. Pollut., 111, 407-415, 2001.

[16] Khan, A., Mir, A.N., Faisal, M.I., Muneer, M., Titanium Dioxide-Mediated Photcatalysed Degradation of Two Herbicide Derivatives Chloridazon and Metribuzin in Aqueous Suspensions. International Journal of Chemical Engineering., 1-8, 2012.

[17] Tutunaru, B., Samide, A., Moanţă, A., Ionescu, C., Tigae, C., Electrochemical Study of Metribuzin Pesticide Degradation on Bismuth Electrode in Aqueous Solution. Int. J. Electrochem. Sci., 10, 223 $-234,2015$. 